\title{
Isolated angiitis of the central nervous system with tumor-like lesion, mimicking brain malignant glioma: a case report and review of the literature
}

\author{
Gan You', Wei Yan', Wei Zhang', Shaowu Li', Guilin Li ${ }^{3}$ and Tao Jiang ${ }^{1 *}$
}

\begin{abstract}
Background: Isolated angiitis of the central nervous system (IACNS) is a rare but severe vascular disease, which could present like an isolated inflammatory lesion on magnetic resonance imaging (MRI). To date, only a few such cases with tumor-like IACNS have been reported.

Case Presentation: A 35-year-old woman presented with headache and left-sided weakness. MRI scans initially mislead us to a diagnosis of glioblastoma (GBM). Surgery was performed. The mass was sub-totally resected. Pathological examination confirmed a cerebral vasculitis. Radiological features, such as disproportionate mass effect, striped hemorrhage and abnormal enhancement of adjacent vessels, could be helpful to distinguish a tumor-like IACNS from a GBM. Single therapy with high doses of steroid did not improve the patient's condition. Combined therapy with prednisolone and cyclophosphamide showed great benefit to the patient. No relapse occurred during the period of 18 months follow-up.

Conclusions: Although a tumor-like IACNS has no established imaging features, a diagnosis of tumor-like IACNS should be suspected when MRI shows inappropriate presentations of a tumor. Greater awareness of this potential manifestation of IACNS may facilitate more prompt diagnosis and treatment.
\end{abstract}

Keywords: primary angiitis, vasculitis, tumor-like lesion, mimicking, glioma

\section{Background}

Isolated angiitis of the central nervous systmen (IACNS) represents a rare and poorly understood form of vascular inflammatory disease restricted to the brain and spinal cord. An average annual incidence rate of 2.4 cases per 1,000,000 person-years was found by a report from US [1]. Histopathology usually reveals granulomatous inflammation affecting arterioles and small arteries of the parenchyma and/or leptomeninges [2]. Non-specific clinical manifestations and various imaging findings often lead to an incorrect or delayed diagnosis and treatment [3], particularly for an extremely rare form of tumor-like lesion. In this report, we describe a woman with tumor-like IACNS that was initially mistaken for glioblastoma (GBM).

\footnotetext{
* Correspondence: taojiang1964@yahoo.com.cn

'Department of Neurosurgery, Beijing Tiantan Hospital, Capital Medical University, Beijing 100050, China Full list of author information is available at the end of the article
}

\section{Case Presentation}

In April 2008, a 35-year-old woman was admitted to our hospital due to headache and left-sided weakness over the preceding 1 month. The headache was diffuse and did not have a burning or stabbing sensation. The weakness of the left arm and jaw was mild. Her mental status was clear with normal orientation and alertness. It was negative in speech disorder and perceptual disturbance. A review of systems at the time of presentation revealed no additional symptoms, except for mild hypomnesis. There was no history of alcohol or illicit drug use. Toxic exposure history was negative. On neurologic examination, the patient presented moderate weakness on the left side. An equivocal Babinski sign was elicited in the left foot. Findings on physical examination were normal. Admission MRI study of the brain (Figure 1) revealed a tumor-like mass with edema and enhancement, which was initially suspected to be a malignant glioma. The patient subsequently underwent a craniotomy. Because a
C Biomed Central 


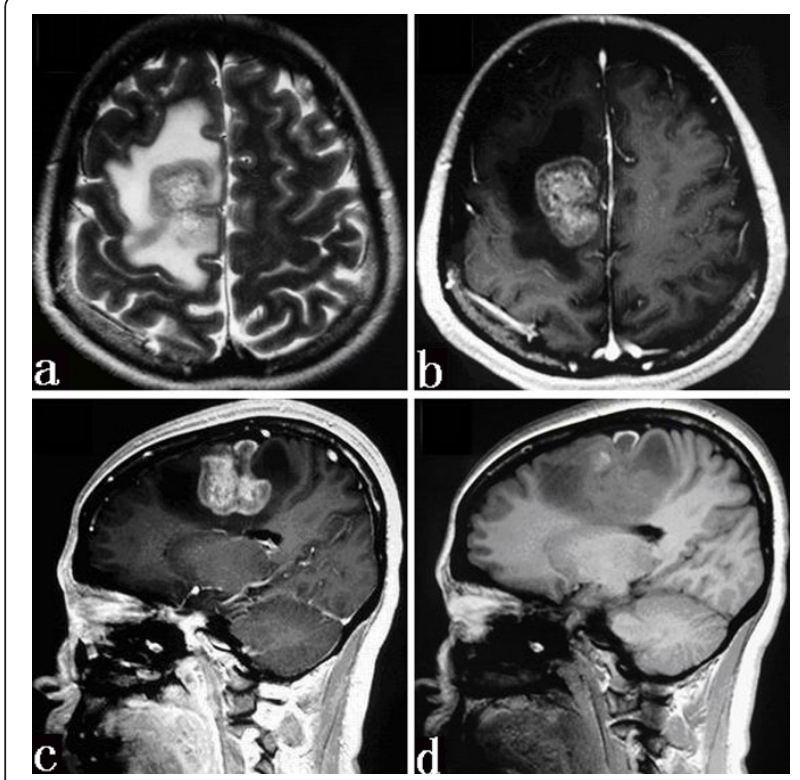

Figure 1 Axial T2-weighted MR image (a) showing a mass with mixed signal intensity and a surrounding edema area. On the T1-weighted image after the administration of contrast material (b and c), the mass has an inhomogeneous enhancement. Sagittal T1weighted MR image without contrast (d) depicting a striped hemorrhage.

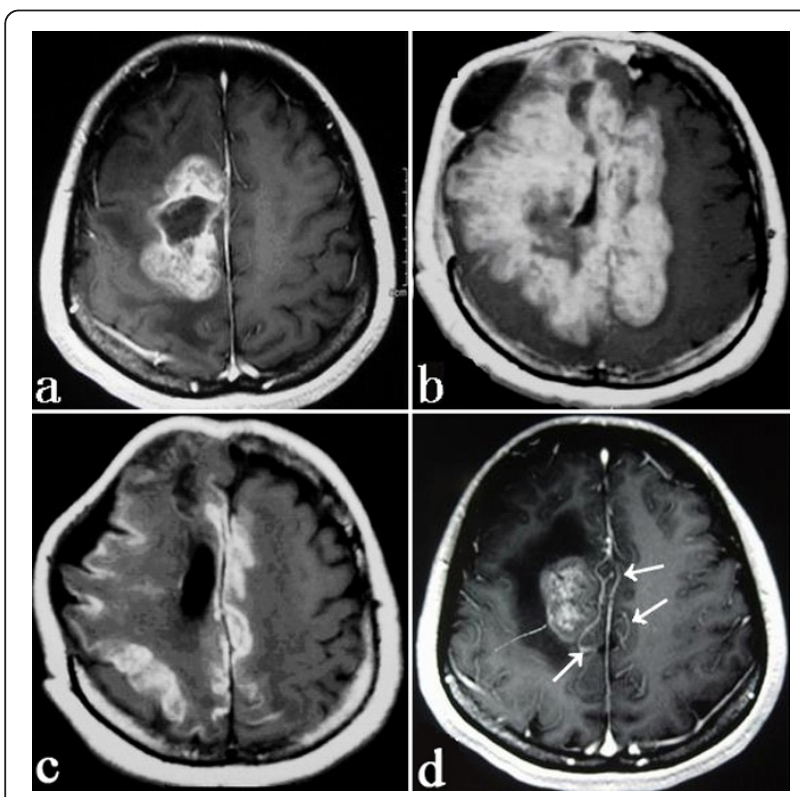

Figure 2 Axial contrast-enhanced MR image showing a partial resection of the lesion. (a) T1-weighted image showing the newly developed hyper-intensity diffusing to the opposite side (b). Great improvement after the treatment (c). T1-weighted MR images with contrast showing some abnormally enhanced vessels (arrows) (d). non-tumoral texture was palpated by the surgeon, the lesion was subtotally resectted (Figure 2a). Intraoperative pathologic examination showed no tumor cells but inflammatory cells and necrosis, which confirmed the surgeon's judgment. The patient's blood and cerebrospinal fluid (CFS) samples were collected at the end of operation. Concerned of a possible diagnosis of multiple sclerosis, a 3-day high-dose (1,000 mg daily) pulse therapy of methylprednisolone was initiated. Two weeks after surgery, the first postoperative MRI showed bilateral hyper-intensity in the frontal and parietal sub-cortex (Figure 2b). Results of the serum and CSF tests were as follows: Routine blood tests, as well as blood rheumatologic tests, blood immunoglobulins, blood antinuclear antibodies, antiphospholipid antibodies, and antineutrophil cytoplasmic antibody, were normal. Analysis of the CSF showed the increases of total protein $(170 \mathrm{mg} / \mathrm{dl}$; normal $<45 \mathrm{mg} / \mathrm{dl})$ and myelin basic protein (MBP) $(2.23 \mathrm{nmol} /$ l; normal $<0.55 \mathrm{nmol} / \mathrm{l})$. The immunoglobulin (Ig) $\mathrm{G}$ in CSF index was remarkably increased $(20.5 \mathrm{mg} / \mathrm{dl}$; normal $<6 \mathrm{mg} / \mathrm{dl}$ ). Oligoclonal bands (OB) were negative. No marked increase in antiviral titers in the serum or CSF was observed. With great interests, we carefully rechecked all the paraffin sections of the mass, and a big surprise for us, we eventually found the evidence for cerebral vasculitis (Figure 3). Combined therapy with steroid and immunosuppressant was initiated immediately after the diagnosis as follows: intravenous prednisolone 1000 $\mathrm{mg} / \mathrm{d}$ for 3 days, $500 \mathrm{mg} / \mathrm{d}$ for 3 days, $250 \mathrm{mg} / \mathrm{d}$ for 3 days, $125 \mathrm{mg} / \mathrm{d}$ for 3 days, followed by oral prednisolone $60 \mathrm{mg} / \mathrm{d}$ and cyclophosphamide $125 \mathrm{mg} / \mathrm{d}$ for 2 weeks. Six weeks after surgery, the patient's neurological symptoms gradually disappeared and repeated MRI confirmed remarkable improvement of affected brain (Figure 2c).

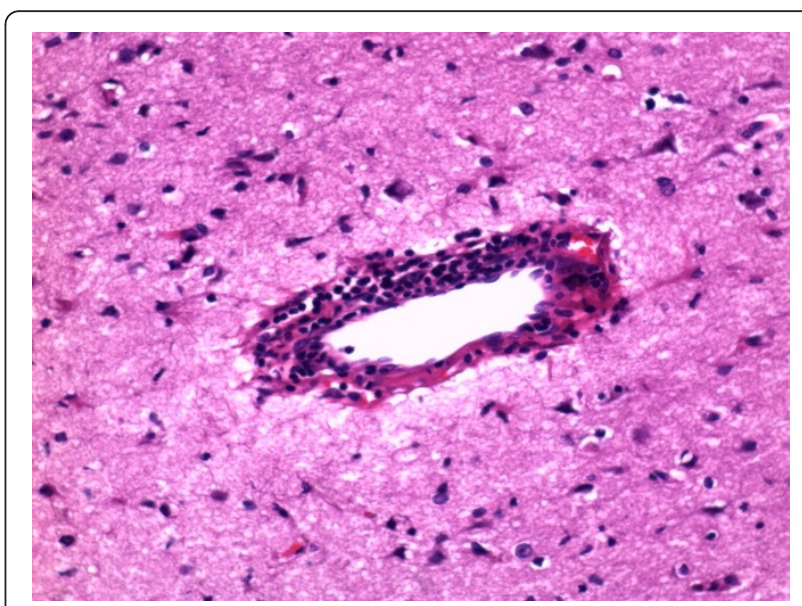

Figure 3 Pathologic findings showing massive perivascular lymphocyte cuffing (HE stain, $\times 200$ ). 
She was discharged on a tapered dose of prednisolone and cyclophosphamide, and no relapse occurred during an 18-month follow-up.

\section{Discussion}

IACNS, which was first reported as early as 1952 [4], can occur mostly when patients are 30 to 50 years old [3]. To date, a few such cases with tumor-like IACNS have been reported. Its etiology and pathogenesis are still unknown. It is very possible that viral infections initiate the inflammatory process that somehow becomes self-sustaining [5]. It is also speculated that there may be a genetic predisposition in certain individuals leading to an enhanced risk of a vasculitic process when there is an exposure to a particular antigen that "sets off" the immune system [6].

Clinical onset of IACNS is usually subacute, but it can have acute onset with rapid progress within a few days or weeks. The most frequent clinical manifestations at presentation are headache, altered cognition, hemiparesis or persistent neurological deficit or stroke [1]. Subarachnoid hemorrhage could be the first presentation of IACNS [7]. Less common complaints are aphasia, transient ischemic attack, ataxia, dysphasia, nausea or vomiting, loss of memory, seizure disorder, dyslalia, hypomnesia and paralysis. Interestingly, there have been so far two case studies reporting psychiatric symptoms in two patients with IACNS $[8,9]$. But all the symptoms above-mentioned are non-specific for diagnosis.

With regard to imaging characteristics, IACNS always presents a challenge in radiographic diagnosis. Its MRI findings are highly variable, ranging from multiple irregular white matter changes to intracerebral hemorrhages $[7,10]$. The present case demonstrated a large monofocal lesion with disproportionate mass effect, striped hemorrhage, and abnormal enhancement of adjacent vessels (Figure 2d) on MRI. Possibly some main artery and its branches were involved. So, given a tumor-like lesion with these characteristics on neuroimages, we suggest an IACNS should be considered in the differential diagnosis. This would be an important lesson learnt from this case. Several studies $[3,11,12]$ to some extent emphasized the similar viewpoints. In addition, Campi et al. [13] indicated that VRPVS (dilatation of VirchowRobin perivascular spaces), which signify severe but reversible perivascular inflammation causing blood-brain barrier disruption and injury of surrounding white matter, might likely be specific for vasculitis. Unfortunately, this did not show up in this case.

Moore [14] recommended a widely received standard for the diagnosis of IACNS in which he emphasized the importance of cerebral angiography and biopsy based on careful exclusion of other diseases. When cerebral angiography shows stenosis or occlusion of the cerebral vessels, brain biopsy should be performed [15]. However, the classic appearance of alternating narrowing and dilatation is not completely specific and has been observed in only $25 \%$ of patients with IACNS; the angiogram is normal in up to $40 \%$ of pathologically documented cases [16]. It should be individualized to make a differential diagnosis $[3,14,17]$. IACNS may clinically mimic encephalitis, multiple sclerosis, acute disseminated encephalomyelitis, myelinoclastic diffuse sclerosis, cerebral infarction, leukoencephalopathy or other brain tumors, when they present isolated lesions on MRI. It may be difficult to differentiate between IACNS and demyelinating or inflammatory diseases [18-20], because of similar symptoms, clinical exam and laboratory findings. Besides, it is also not easy to differentiate between IACNS and lymphoma when there are multiple-enhancing lesions with vasogenic edema on neuroimagines. Against the above, only biopsy allows a definite diagnosis. But sometimes, a single isolated negative biopsy does not necessarily exclude IACNS $[21,22]$ or secondary CNS vasculitis. In that case, empirical treatment should be administrated.

No clinical trials have been performed in patients with IACNS, nor is it possible to draw firm conclusions from the current study because of the non-standardized nature of the treatment protocols used in these cases. Fountain et al. [23] reported a case with IACNS controlled by cyclophosphamide alone, while Carandang $\mathrm{C}$ and Grant A reported a female patient with IACNS responding to steroids alone [9]. But Barron et al. [24] indicated that steroid therapy alone failed to improve the condition. This finding is consistent with ours. Others recommended combination therapy consisting of prednisone and cyclophosphamide for at least 1 year [25,26]. In addition, Salvarani et al. [27] indicated that patients with IACNS which also have vascular deposits of $\beta$-peptide generally respond well to immunosuppressive treatment. However, the present case demonstrated good effects of immunosuppressant to patient without indication of amyloid protein deposition. Currently, most authors consent to combined and long-term therapy in treating IACNS. It is strongly suggested $[1,28]$ that immunosuppressive therapy should be judiciously employed in patients with IACNS, based on the clinical features (any co-morbid conditions, the potential for neurological recovery, et al). In our case, the initial pulse therapy with steroid failed to prevent the rapid progress of vasculitis. Only combined therapy with cyclophosphamide improved her condition dramatically.

The prognosis of IACNS varies greatly and could be from months to years $[1,11,17]$. Untreated IACNS usually causes fatal outcome. Greater awareness of these potential manifestations of IACNS may facilitate more accurate diagnosis and prompt treatment. 


\section{Conclusions}

In conclusion, we suggest that correct diagnosis and appropriate treatment of tumor-like IACNS should be essential for prognosis. When the MRI shows a tumorlike subcortical lesion with disproportionate mass effect, and/or striped hemorrhage and/or abnormal enhancement of adjacent vessels, an IACNS should be include in the differential diagnosis. When high doses of steroids show no effect to the patient with tumor-like IACNS, combined treatment with cyclophosphamide followed by long term oral therapy is recommended.

\section{Consent}

Written informed consent was obtained from the patient for publication of this Case report and any accompanying images. A copy of the written consent is available for review by the Editor-in-Chief of this journal.

\section{Author details \\ 'Department of Neurosurgery, Beijing Tiantan Hospital, Capital Medical University, Beijing 100050, China. ${ }^{2}$ Department of Neuroradiology, Beijing Tiantan Hospital, Capital Medical University, Beijing 100050, China. \\ ${ }^{3}$ Department of Pathology, Beijing Neurosurgical Institute, Beijing 100050, China}

\section{Authors' contributions}

GY wrote the initial draft. WY drew the pictures. WZ revised the draft. SL described the MRI features. GL provided the pathological diagnosis. TJ was the surgeon and gave the final approval of the version to be published.

\section{Competing interests}

The authors declare that they have no competing interests.

Received: 18 May 2011 Accepted: 26 August 2011

Published: 26 August 2011

\section{References}

1. Salvarani C, Brown RD Jr, Calamia KT, Christianson TJ, Weigand SD, Miller DV, Giannini C, Meschia JF, Huston J, Hunder GG: Primary central nervous system vasculitis: analysis of 101 patients. Ann Neurol 2007, 62:442-451.

2. MacLaren K, Gillespie J, Shrestha S, Neary D, Ballardie FW: Primary angiitis of the central nervous system: emerging variants. Q J Med 2005, 98:643-654.

3. Markus K, Peter B: Primary central nervous system vasculitis: clinical experiences with 21 new European cases. Rheumatol Int 2009, 31:463-472.

4. Newman W, Wolf A: Non-infectious granulomatous angiitis involving the central nervous system. Trans Am Neurol Assoc 1952, 56:114-117.

5. Iwase T, Ojika K, Mitake S, Katada E, Katano H, Mase M, Yoshida S, Ueda R: Involvement of CD45RO+ T lymphocyte infiltration in a patient with primary angiitis of the central nervous system restricted to small vessels. Eur Neurol 2001, 45:184-185.

6. Kelley RE: CNS vasculitis. Front Biosci 2004, 9:946-955.

7. Kumar R, Wijdicks EF, Brown RD Jr, Parisi JE, Hammond CA: Isolated angiitis of the CNS presenting as subarachnoid haemorrhage. I Neurol Neurosurg Psychiatry 1997, 62:649-651.

8. Hocaoglu C, Tan M: Isolated angiitis of the central nervous system: a case presented with atypical psychiatric symptoms. Prog Neuropsychopharmacol Biol Psychiatry 2005, 29:627-631.

9. Carandang C, Grant A: Delirium and Isolated Angiitis of the Central Nervous System: a Case Report and Review. CNS Spectrums 2008, 13:209-213.
10. Ozawa T, Sasaki O, Sorimachi T, Tanaka R: Primary angiitis of the central nervous system: report of two cases and review of the literature. Neurosurgery 1995, 36:173-179.

11. Lee Y, Kim JH, Kim E, Park SH, Yim YJ, Sohn CH, Chang KH: Tumormimicking primary angiitis of the central nervous system: initial and follow-up MR features. Neuroradiology 2009, 51:651-659.

12. Ay H, Sahin G, Saatci I, Söylemezoğlu F, Saribaş O: Primary Angiitis of the Central Nervous System and Silent Cortical Hemorrhages. AJNR Am J Neuroradiol 2002, 23:1561-1563

13. Campi A, Benndorf G, Filippi M, Reganati P, Martinelli V, Terreni MR: Primary angiitis of the central nervous system: serial MRI of brain and spinal cord. Neuroradiology 2001, 43:599-607.

14. Moore PM: Diagnosis and management of isolated angiitis of the central nervous system. Neurology 1989, 39:167-173.

15. Younger DS: Vasculitis of the nervous system. Curr Opin Neurol 2004 17:317-336.

16. Calabrese LH, Furlan AJ, Gragg LA, Ropos TJ: Primary angiitis of the central nervous system: diagnostic criteria and clinical approach. Cleve Clin J Med 1992, 59:293-306.

17. Scolding NJ, Wilson H, Hohlfeld R, Polman C, Leite I, Gilhus N: The recognition, diagnosis and management of cerebral vasculitis: a European survey. Eur J Neurol 2002, 9:343-347.

18. Tan HM, Chan LL, Chuah KL, Goh NS, Tang KK: Monophasic, solitary tumefactive demyelinating lesion: neuroimaging features and neuropathological diagnosis. Br J Radiol 2004, 77:153-156.

19. Mandrioli J, Ficarra G, Callari G, Sola P, Merelli E: Monofocal acute large demyelinating lesion mimicking brain glioma. Neurol Sci 2004, 25 S386-388.

20. Engelter ST, Rueegg S, Kirsch EC, Fluri F, Probst A, Steck AJ, Lyrer PA: CADASIL mimicking primary angiitis of the central nervous system. Arch Neurol 2002, 59:1480-1483.

21. Dawson TM, Starkebaum G: Isolated central nervous system vasculitis associated with hepatitis C infection. J Rheumatol 1999, 26:2273-2276.

22. Alreshaid AA, Powers WJ: Prognosis of patients with suspected primary CNS angiitis and negative brain biopsy. Neurology 2003, 61:831-833.

23. Fountain NB, Lopes MB: Control of primary angiitis of the CNS associated with cerebral amyloid angiopathy by cyclophosphamide alone. Neurology 1999, 52:660-662.

24. Barron TF, Ostrov BE, Zimmerman RA, Packer RJ: Isolated angiitis of CNS: treatment with pulse cyclophosphamide. Pediatr Neurol 1993, 9:73-75.

25. Guillevin L, Ramanoelina J, Mahr A: Central nervous system and systemic vasculitides. Rev Neurol (Paris) 2002, 158:907-913.

26. Kluck JJ, ter Berg JW, van der Laan RT, Krijne-Kubat B: Two patients with isolated vasculitis of the central nervous system. Ned Tijdschr Geneeskd 2002, 146:482-486.

27. Salvarani C, Brown RD Jr, Calamia KT, Christianson TJ, Huston J, Meschia JF, Giannini C, Miller DV, Hunder GG: Primary central nervous system vasculitis: comparison of patients with and without cerebral amyloid angiopathy. Rheumatology 2008, 47:1671-1677.

28. Molloy ES, Singhal AB, Calabrese LH: Tumour-like mass lesion: an underrecognised presentation of primary angiitis of the central nervous system. Ann Rheum Dis 2008, 67:1732-1735.

doi:10.1186/1477-7819-9-97

Cite this article as: You et al:: Isolated angiitis of the central nervous system with tumor-like lesion, mimicking brain malignant glioma: a case report and review of the literature. World Journal of Surgical Oncology 2011 9:97. 